\title{
Neurochirurgie et Créativité
}

\author{
Christian Phéline
}

Can. J. Neurol. Sci. 1990; 17:49-52

Les mots de Neurochirurgie et de Créativité, semblent à priori antagonistes. D'une part, le monde de cumul, d'érudition, d'exactitude, de codification de la science neurochirurgicale, et d'autre part, celui de la fantaisie et de la liberté de l'improvisation et de l'aléatoire.

Pourtant, devant la stagnation de certaines «récupérations» cérébrales, nous avons senti la nécéssité d'une démarche supplémentaire affranchie des normes traditionnelles.

C'est qu'il s'agit, en face d'une chronicité désespérante, non plus de réparer l'organe, mais de rétablir les repères, les circuits, les motivations, les significations que le cerveau n'est plus capable d'intégrer. Plus que dans toute autre pathologie, à la lésion cérébrale grave, il faut opposer une attitude thérapeutique créative.

Les expériences de Rozensweig sur la plasticité cérébrale ont bien démontré l'influence d'un environnement enrichi sur le réveil et la récupération des animaux cérébro-lésés. ${ }^{1}$

Dans un service de Neurochirurgie, nous affrontons aussi le problème du délai et de la qualité de récupération d'une lésion cérébrale. Nous essayons d'influencer les possibilités de plasticité cérébrale. Nous essayons d'enrichir le milieu mais si le rat de laboratoire se contente de quelques jouets variés, d'une amélioration de sa cage, de la présence de congénères, combien plus grande est l'exigence de l'homme dans sa complexité. Son univers enrichi peut-il être standardisé ? Toute approche écologique du cérébro-lésé humain demande un investissement en créativité.

\section{la Creation Permanente de soi Caracteristique de L'ETRE CONSCIENT}

L'être conscient est en création permanente de sa personne. Pour H. Ey, il est en possession d'une représentation du monde et de lui même indispensable à l'action et mise à jour à travers un dialogue perpétuel avec son corps, avec le monde, avec son passé et avec son inconscient. ${ }^{2}$

Si on parle en terme d'information, l'homme est doué, grâce à sa structure nerveuse remarquable par ses redondances multiples et sa fiabilité, d'une possibilité de trier l'information et de se l'assimiler de telle façon qu'elle participe à sa structure. C'est ce que Henri Atlan appelle l'auto-organisation. Nous sommes des systèmes auto-organisateurs capables d'extraire du bruit exterieur les messages compatibles et de rejeter ceux qui risquent de déborder la flexibilité de notre organisation personnelle. ${ }^{3}$

Nous voici donc traversés perpétuellement par un flux d'informations et d'énergie en provenance de notre environnement, énergie et informations étant indispensables au maintien de notre personne.

On peut dire qu'il existe un phénomène de création de soi à partir des informations reçues et cette créativité de soi est une fonction basale de l'individu.

Comment cette fonction basale se modifie-t-elle face à la maladie ? Balint situe dans la "zone de création" "à la foisles phases précoces de l'entrée dans la maladie physique ou psychique et la guérison spontanée d'une maladie". ${ }^{4}$ L'application à la neurochirurgie du concept de créativité nous amène à considérer successivement: créativité du malade neurochirurgical; créativité du soignant et interaction des deux créativités.

\section{Le Malade Neurochirukgical}

C'est l'étude du coma prolongé qui nous a amenés à plusieurs années de réflexion sur les aspects spécifiques de la maladie cérébrale : au cours du coma, il existe une interruption du courant d'information que lie l'individu à sa structure extérieure il y a perte du dialogue avec l'environnement, suspension des mécanismes créateurs de l'identité, rôle iatrogène de l'hôpital.

En effet la personne humaine est quelquechose de très fragile, elle se construit à partir des messages venus du corps et du monde extérieur. Dans le coma au contraire, il y a interruption du vécu, un véritable trou dans l'histoire personnelle, interruption de ce flux permanent d'informations et d'énergie en provenance de l'environnement et de la structure de vie habituelle. La permanence de la personne n'est plus assurée.

Pour des raisons organiques, tout d'abord, il est certain que les circuits habituels sont perturbés, sur le plan sensoriel, sur le plan mnésique, sur le plan de l'efficacité du geste.

Pour des raisons fonctionnelles également, car le situation de confinement prolongé en milieu hospitalier réalise pour l'individu la situation d'un animal de laboratoire : contraint, contrôlé de l'extérieur, incapable d'agir sur son environnement, de mani-

Cette communication a été présentée à la réunion de Neurochirurgie de l'Hôpital Notre Dame - Montreal, le 10.06.88, à l'occasion du 40 ème anniversaire de la création du service.

Du Centre Hospitalier régional d'Orléans, France

Reçu le 3 mai 1989. Accepté le 22 aôut 1989

Tirés à part: Docteur C. Pheline, Chef de Service de Neurochirurgie, C.H.R. La Source B.P., 6709-45067 Orléans Cédex 2, France 
fester son agressivité ou son initiative, situation hautement anormale qui, à travers l'intensité des stimulations non significatives et la privation de gratification et de repères habituels, pourrait aboutir à ce que Kurt Golstein a décrit comme la situation de catastrophe, à la fermeture des sens au refus du contact avec l'extérieur, au repli sur soi-même, analogue à la mort simulée des animaux.5

Le phénomène le plus important, au cours de la lésion cérébrale grave est peut être justement l'interruption de la fonction de créativité, fonction fondamentale à l'origine du maintien de l'individu dans son sentiment d'existence.

Dans les état d'insuffisance fonctionnelle cérébrale manifestée par le coma il y a donc interruption de cette entité que nous savons reconnaitre chez chaque être humain : la personne.

Cependant, dans le corps, dans la structure nerveuse, quelque détériorée qu'elle soit, il y a persistance de l'organisation initiale, originale, celle qui préexistait à la maladie cérébrale. Elle permettait à chaque individu, d'accorder un sens à l'évènement, de décoder l'information selon ses propres critères, d'élaborer une stratégie d'adaptation convenable en fonction du vécu antérieur et de l'expérience, en somme d'accorder une valeur affective et éthique à la situation.

Si l'on espère aider les phénomènes de plasticité cérébrale, il est certain que ce sera en utilisant la même direction, les mêmes schemas, les mêmes patterns que ceux qui préexistaient chez notre patient avant son accident pathologique. La prise en charge d'un malade neurochirurgical sévèrement cérébro-lésé comporte un certain nombre de démarches de créativité spécifiques de l'individu auquel on s'adresse. S'il s'agissait d'un enfant ou d'un vieillard ou encore du client d'une entreprise, cette façon de considérer les choses paraîtrait tout à fait justifiée. Ce qui est original c'est d'appliquer à la neurochirurgie des techniques qui ont pu être éprouvées dans d'autres domaines. Finalement, il s'agit d'ouvrir une spécialité extrêmement protégée aux acquisitions de l'écologie, l'éthologie, l'étude des motivations en général, les conceptions systémiques de l'être humain. Si ces differents emprûnts sont difficiles à appliquer, c'est qu'ils réclament de la part du soignant la prise de risque évidents, la première des audaces consistant à prendre en charge de façon absolue, d'assumer ce type de blessé en oubliant ses propres préoccupations et en ayant achevé la besogne neurochirurgicale normale. L'investissement nécessité par ce type de patient risque d'amener le soignant très loin et de déboucher sur beaucoup plus souvent des désillusions que des succès évidents. ${ }^{6}$

\section{Creativite du Soignant}

Cette réflexion est le résultat d'une approche multi-disciplinaire commune à l'équipe de Neuropsychologie, Psychologues, Orthophonistes, Kinésithérapeutes et personnel infirmier. Le souci de rétablir l'identité du malade a fait apparaître peu à peu la nécessité des quatre démarches de créativité que nous allons exposer:

- Première démarche: Création d' une modèle du blessé.

S'il est facile à créer, pour le rat de laboratoire, l'univers enrichi est plus difficile à définir chez l'homme et doit être étudié à la carte pour chaque patient déterminé.

Aussi, avons-nous trouvé utile de réaliser dès le début de l'hôpitalisation de notre blessé, une enquête approfondie, de façon à produire un modèle le plus exact possible de la psychologie de ce malade, de ses goûts, de ses habitudes, de façon à pouvoir imaginer quels seraient ses besoins véritables et d'y apporter une réponse, comme s'il était capable de les exprimer lui-même. ${ }^{7}$ Les entretiens immédiats avec la famille peuvent bien sûr nous renseigner sur le blessé que nous traitons, mais est-il possible aux proches d'être objectifs dans de telles circonstances ? En réalité, c'est la visite du domicile et du lieu de travail qui nous a fourni les meilleurs renseignements sur la structure dans laquelle évolue habituellement le blessé. Nous avons retiré de ces visites une moisson d'indices concernant les lieux, les objets, les êtres qui entourent habituellement le malade et peu à peu se définit une espèce d'image psychologique qui vient compléter ce que nous savons du corps inanimé que nous réanimons.

Ce modèle du malade est aussi discutable qu'une oeuvre d'art : imparfaite, néanmoins l'oeuvre d'art est un moyen de provoquer et amène à partager une émotion, elle sollicite un échange. De la même façon, lorsque nous rentrons du domicile, nous échangeons avec le reste de l'équipe notre impression. Nous exposons dans la chambre les objets que nous avons ramenés et qui sont les témoins de la personne du blessé de sorte que dans un effort de créativité, nous obtenons que ce malade inanimé reste une personne à part entière dans l'esprit des soignants et de la famille et que de cette façon ses droits et sa place restent préservés dans la société, au sein de la famille, au lieu de travail, comme si nous étions dépositaires et garants du malade qui nous est confié, et ceci non seulement dans sa dimension physique mais en tant que personne morale.

Ce qu l'on obtient de la sorte c'est que l'attitude du personnel devienne plus déférente et respectueuse. C'est là la première amélioration que nous apportons à l'environnement : nous espérons qu'il devienne plus compatible, plus pertinent pour ce blessé et nous allons continuer d'oeuvrer de façon à ce que le milieu que nous créons soit tel que cet individu puisse s'y reconnaître un jour.

- Deuxième démarche : une triangulation dynamique

L'équipe soignante et la famille doivent être habitées d'une volonté de recréation de la personne en suspens. Autour de cette personne s'établit un circuit d'informations et de collaborations qui engage absolument toutes les personnes concernées.

Les rôles à l'intérieur de cette triangulation dynamique famille-soignant-malade sont répartis différemment suivant les moments de la journée et suivant le temps de l'évolution. En effet, le malade va reprendre en charge peu à peu ses autonomies. Le soignant de son côté introduit la famille aux soins et lui enseigne les différentes techniques. Il y a peu à peu transfert des responsabilités et un retour précoce au domicile est ainsi facilité. De plus, la famille qui est engagée dans la lutte ne s'éloigne pas de son patient. Ce qui doit être évident pour tous, c'est que c'est l'agenda du malade qui commande et non pas celui du soignant ou de la famille. C'est le malade qui va choisir le temps opportun pour lui au cours de sa 
réorganisation pour s'éveiller, pour s'agiter, exécuter certains ordres écouter le discours et enfin participer à l'échange.

L'objectif est donc de proposer au malade et non pas de lui imposer une quelconque information.

Le patient, dégagé temporairement du contrôle de ses superstructures corticales se comporte comme s'il était dans un état naissant, ouvert à certains apprentissages :

Un état fait d'émotion primitive et déraisonnée, probablement proche de celui qui était caractéristique de nos ancêtres préhistoriques avant l'installation du langage. On doit savoir combien l'attente et l'anxiété de ce type de blessés sont profonds. De même que le mystique attend un signe de son Dieu au cours de la méditation, de même le comateux qui s'éveille attend tout de son prochain, un encouragement, une communication, une presence? Il est en tout cas souhaitable d'observer et de valoriser un éventuel choix du blessé qui préfèrera manger, être entouré, être traité par tel ou tel élement du personnel.

- Troisième démarche : Un milieu et une relation adaptés.

Alors que chez l'animal on parle de stimulation et d'environnement, chez l'homme il faudrait penser plutôt situation et évènements. En effet, chacun d'entre nous ne represente que ce que le monde extérieur l'amène à être et si nous essayons de créer un environnement qui convienne particulièrement à chaque blessé, ce milieu se doit d'être mobile, stimulant, exigeant, sans excès.

Winnicott a bien défini pour l'enfant l'univers transitionnel, univers intermédiaire que nous devons également créer entre le monde nécéssaire de la réanimation et le monde exigeant et répulsif de la réalité. ${ }^{8}$

Pour que le malade se regroupe et organise ses résistances, pour qu'il accepte d'affronter les contraintes de la relation avec l'autre et du retour au monde, il faut que ceci se passe dans une atmosphère gratifiante, une atmosphère de jeu et de plaisir et ceci va nous mettre en alerte et exiger de nous une créativité permanente. ${ }^{8}$ En effet, à chaque stade de l'évolution, il va falloir organiser cette espècede retrouvaille avec la vie et il faudra emprunter sans cesse à tout ce qui nous est proposé pour composer à la demande l'évènement et la situation du jour : famille, locaux, nature, domicile, petites cérémonies quotidiennes du bain, de la visite, du repas, de la sortie au jardin.

Ce qui est difficile à declencher chez ce malade c'est la motivation alors qu'il se réfugie dans l'inertie ou au contraire dans des réactions primitives avec aggressivité visà-vis de toute frustration. 9,10

Pour le motiver, on est obligé de l'exposer aux intempéries, de supprimer tous les catheters, tubes, drip feeding, etc. . . . qui assurent bien sûr, la survie, mais qui en même temps l'empêchent de ressentir les besoins et les désirs qui forment le rythme de nos journées depuis la naissance (sensation de vide plein).

Lorsqu'un «plateau» apparaît dans le progès du malade, on doit se dire que l'environnement proposé n'est plus compatible et qu'il y a lieu de le modifier. C'est le moment qui est choisi pour la sortie au jardin ou pour le transport bref au domicile ou pour faciliter ou encore éviter la venue de tel ou tel membre de la famille. Ainsi le soignant a le devoir de maîtriser dans une certaine mesure les données de l'environnement.

La communication s'établit sur un plan émotionnel. Il y aurait toute une étude sémiotique à réaliser sur les possibilités d'échange non verbal avec ce type de patient, ce qui est très curieux, c'est que le jeune enfant ou l'animal ou encore l'être très frustre sont capables d'établir une communication avec l'adulte évolué, mais le soignant el certains membres de la famille ne peuvent la réaliser parce que étant protégées par toute une carapace de comportements acquis, ces dernières personnes sont incapables de s'investir dans une situation tout à fait inattendue. ${ }^{11}$

- Quatrième démarche : le soignant doit créer sa propre aptitude à la relation.

Pour accéder à un certain Niveau de communication inter-personnelle, il est nécessaire que le soignant échappe à ses à priori culturels et à ses lignes de défense personnelles. II lui faut, dans une certaine mesure, faire le vide et les disciplines de méditation orientales ou autres sont quelquefois pour certaines personnalités d'un grand secours (Yoga, Zen, Training autogène, etc. ...).

Le soignant doit transgresser ses instincts habituels. II va à l'encontre du sentiment de sécurité qui consiste à maintenir les différents appareillages de réanimation qui privent le malade de la sensation de vide-plein (poumons, vessie, intestins) nécessaires au retour des besoins physiologiques. Le patient doit accepter la présence de la famille qui n'est pas toujours facile à tolérer dans le parcours technique.

Le soignant doit paradoxalement laisser aller son émotion car c'est par l'émotion que la communication s'établit avec le blessé. Elle doit être objectivée.

Ceci est donné aux membres les plus humbles du personnel qui, ignorant tout de la condition organique dans laquelle se trouve situé le système nerveux de blessé, s'adressent à lui comme s'il était parfaitement conscient, parfaitement lucide et en possession de son statut social habituel.

\section{L'interaction des Creativités}

Un univers incompatible peut amener chez certains traumatisés crâniens dont la personnalité ne se regroupe pas spontanément au sortir du coma, une véritable réaction psychopathologique marquée par la disparition de toute énergie de créativité personnelle.

C'est l'isolement, la panique, le désarroi qui aboutissent à un repli, à une défaite des forces profondes de l'individu.

Face à cette maladie, il y a possibilité de faire figurer en miroir la créativité du soignant. Tel un moniteur, le soignant offre en exemple son appétit de vivre. C'est ainsi que la volonté de guérison est sollicitée au cours de cette situation que est analogue à celle de la fin de la vie comme l'a montré B. Hagglund. 12 Nous avons montré comment il est possible d'introduire dans un service de Neurochirurgie une approche particulière supplémentaire des états de dévastation de la per- 
sonne, la notion de créativité est le dénominateur commun de tous les termes particuliers de cette approche : réalisation précoce d'un modèle du malade, réalisation d'un environnement adapté, création d'une dynamique triangulaire particulière dans la manoeuvre de soins, et enfin développement de la créativité du soignant, face à le créativité dévastée du patient.

Lorsque Cezanne dessine trois pommes sur une assiette, il organise sur sa toile une certaine vérité du monde, il nous la communique en un clin d'oeil. Lorsque Saint Jean de la Croix répète son vers comme une caresse ou juxtapose deux images inattendues, il déclenche sans que l'on sache bien pourquoi le plaisir ou le trouble et nous transporte au plan de la sagesse humaine. Le poète et l'artiste savent laisser parler leurs profondeurs dans leur création. Leur émotion initiale traverse le fatras de notre activité superficielle pour parvenir au plus réel de notre être. Ils ont su dire l'essentiel.

Le médecin sait-il encore être créatif?

\section{Bibliographie}

1. Rosenzweig in Bach Y, Rita P. Recovery of function. Bern : Hanshuber 1980.
2. Ey H. La conscience. PUF.

3. Atlan H. Le principe d'ordre à partir du bruit in 'l'unité de l'homme'. Dans: Morin E, Piattelli M, Palmarini. Tome 2, Points Seuil. Paris 1975.

4. Balint $M$. Le defaut fondamental : aspects therapeutiques de la regression. PBP 1977.

5. Golstein K. La structure de l'organisme. Gallimard NRF 1951.

6. Bereznak KC. The mythic artery the magic of music therapy. Ridgeview Pub Co 1982.

7. Daumezon M. Dans: Maruani C. Ethique et psychiatrie. Privat Cahors 1978.

8. Winnicott DW. Jeu et réalité. Paris : Gallimard 1982.

9. Stephanos $S$. Libinal cathexis and emotional growth in the analytical treatment of psychosomatic patients. Psychoter and Psychosom; 32: 101-111.

10. Bettelheim B. Survivre - Pluriel Ed Laffont 1979.

11. Spitz RA. De la naissance à la parole. Paris : PUF 1968.

12. Hagglund TB. Dying : a psychoanalytical study with special reference to individual creativity and defensive organization. Psychiatria Fennica 1972. 